Published in final edited form as:

Am J Ophthalmol. 2018 September ; 193: 54-61. doi:10.1016/j.ajo.2018.06.003.

\title{
Longitudinal Changes of Fixation Location and Stability Within 12 Months in Stargardt Disease: ProgStar Report No. 12
}

\section{ETIENNE M. SCHÖNBACH,}

Wilmer Eye Institute, Johns Hopkins University, Baltimore, Maryland, USA; Department of Ophthalmology and Visual Sciences, Case Western Reserve University, Cleveland, Ohio, USA

\section{RUPERT W. STRAUSS,}

Wilmer Eye Institute, Johns Hopkins University, Baltimore, Maryland, USA; Department of Ophthalmology, Medical University, Graz, Austria; Moorfields Eye Hospital, London, UK; Department of Ophthalmology, Johannes Kepler University, Linz, Austria

\section{XIANGRONG KONG,}

Wilmer Eye Institute, Johns Hopkins University, Baltimore, Maryland, USA; Department of Biostatistics and Epidemiology, University of Massachusetts-Amherst, Amherst, Massachusetts, USA

\section{BEATRIZ MUÑOZ,}

Wilmer Eye Institute, Johns Hopkins University, Baltimore, Maryland, USA

\section{MOHAMED A. IBRAHIM,}

Wilmer Eye Institute, Johns Hopkins University, Baltimore, Maryland, USA

\section{JANET S. SUNNESS,}

Hoover Low Vision Rehabilitation Services, Greater Baltimore Medical Center, Baltimore, Maryland, USA

DAVID G. BIRCH,

Retina Foundation of the Southwest, Dallas, Texas, USA

\section{GESA-ASTRID HAHN,}

Center for Ophthalmology, Eberhard Karls Universität, Tübingen, Germany

\section{FADI NASSER,}

Center for Ophthalmology, Eberhard Karls Universität, Tübingen, Germany

\section{EBERHART ZRENNER, \\ Center for Ophthalmology, Eberhard Karls Universität, Tübingen, Germany}

\section{SRINIVAS R. SADDA,}

Doheny Eye Institute, UCLA David Geffen School of Medicine, Los Angeles, California, USA

\section{SHEILA K. WEST,}

Inquiries to Hendrik P. N. Scholl, Institute of Molecular and Clinical Ophthalmology Basel (IOB), Mittlere Strasse 91, CH-4031 Basel, Switzerland; Hendrik.Scholl@usb.ch.

A listing of the ProgStar Study Group is included in the Appendix (Supplemental Material available at AJO.com). Supplemental

Material available at AJO.com. 
Wilmer Eye Institute, Johns Hopkins University, Baltimore, Maryland, USA

HENDRIK P.N. SCHOLL

Wilmer Eye Institute, Johns Hopkins University, Baltimore, Maryland, USA; Institute of Molecular and Clinical Ophthalmology Basel (IOB), Basel, Switzerland; Department of Ophthalmology, University of Basel, Basel, Switzerland

\section{PROGSTAR STUDY GROUP}

\section{Abstract}

PURPOSE: To investigate the natural history of Stargardt disease (STGD1) using fixation location and fixation stability.

DESIGN: Multicenter, international, prospective cohort study.

METHODS: Fixation testing was performed using the Nidek MP-1 microperimeter as part of the prospective, multicenter, natural history study on the Progression of Stargardt disease (ProgStar). A total of 238 patients with $A B C A 4$-related STGD1 were enrolled at baseline (bilateral enrollment in $86.6 \%$ ) and underwent repeat testing at months 6 and 12 .

RESULTS: Outcome measures included the distance of the preferred retinal locus from the fovea (PRL) and the bivariate contour ellipse area (BCEA). After 12 months of follow-up, the change in the eccentricity of the PRL from the anatomic fovea was -0.0014 degrees $(95 \%$ confidence interval $[\mathrm{CI}],-0.27$ degrees, 0.27 degrees; $P=.99$ ). The deterioration in the stability of fixation as expressed by a larger BCEA encompassing 1 standard deviation of all fixation points was 1.21 degrees squared $\left(\mathrm{deg}^{2}\right)\left(95 \% \mathrm{CI},-1.23 \mathrm{deg}^{2}, 3.65 \mathrm{deg}^{2} ; P=.33\right)$. Eyes with increases and decreases in PRL eccentricity and/or BCEA values were observed.

CONCLUSIONS: Our observations point to the complexity of fixation parameters. The association of increasingly eccentric and unstable fixation with longer disease duration that is typically found in cross-sectional studies may be countered within individual patients by poorly understood processes like neuronal adaptation. Nevertheless, fixation parameters may serve as useful secondary outcome parameters in selected cases and for counseling patients to explain changes to their visual functionality.

THE MOST COMMON TYPE OF JUVENILE MACULAR degeneration is Stargardt disease (STGD1; OMIM: 248200). ${ }^{1,2}$ Affected individuals start to develop progressive decline in best-corrected visual acuity (BCVA), often within the first or second decades of life. ${ }^{3}$ Currently, no treatment to preserve or restore vision is available for STGD1 patients. New therapeutic approaches, such as pharmacotherapy, gene therapy, stem cell therapy, retinal prostheses, and optogenetics are being developed; some of them are already being investigated in clinical trials. ${ }^{2,4,5}$ Outcome parameters for STGD1 require special considerations with respect to the centrifugal progression of atrophy in STGD1 (initially affecting the macula before the periphery). ${ }^{4}$

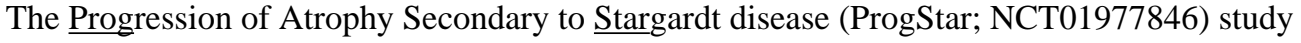
investigates structural and functional outcome measures in the natural history of STGD1. ${ }^{6}$ We recently showed that fundus autofluorescence (FAF) as an anatomic measure may serve as a monitoring tool for clinical trials for STGD1. ${ }^{7,8}$ BCVA, which has been the primary 
functional outcome measure for most treatment trials for retinal diseases in the past, is not a sensitive parameter for STGD1 and may be reduced early in the course of the disease owing to foveal involvement. ${ }^{7,9,10}$ Fundus-tracking microperimetry can establish structure-function correlation between topographic locations of the macula in STGD1, ${ }^{11}$ and it can also characterize an eye's fixation behavior. ${ }^{12}$ Prior research has shown that fixation stability correlates with reading speed ${ }^{13-16}$ and visual search ability ${ }^{17}$ in patients with macular disease. We recently demonstrated the complex relationship of fixation location and stability with BCVA in STGD1, ${ }^{12}$ but little data on the progression of fixation parameters in STGD1 are available to date. ${ }^{18}$

The purpose of this research is to investigate longitudinal changes of fixation location and stability in a large cohort of molecularly confirmed STGD1 patients from the ProgStar study. These parameters may prove useful to describe the disease progression.

\section{METHODS}

MICROPERIMETRY TESTING WAS PERFORMED AS PART OF the ProgStar study, a prospective natural history study on the progression of STGD1 (cohort study). ICH GCP Guidelines, the applicable regulatory requirements, and the current Declaration of Helsinki were followed. All participants provided written informed consent prior to enrollment into the study. ProgStar is in compliance with HIPAA and was approved by the Western Institutional Review Board (IRB), the local IRBs, and the Human Research Protection Office of the U.S. Army Medical Research \& Materiel Command prior to enrollment of the first patient.

\section{PATIENT ELIGIBILITY AND EXCLUSION CRITERIA:}

ProgStar report no. $1^{6}$ describes the design, organization, and inclusion and exclusion criteria in detail. Key inclusion criteria relevant to this report include a minimum age (aged $\geq$ 6 years) and the presence of $\geq 2$ pathogenic mutations in the $A B C A 4$ gene or 1 pathogenic mutation with a typical STGD1 phenotype. At least 1 well-demarcated area of atrophy as visualized by FAF with a minimum diameter of $300 \mu \mathrm{m}$ was required for inclusion and all lesions together had to add to less than or equal to $12 \mathrm{~mm}^{2}$ (equivalent to no more than 5 disc areas in at least 1 eye) in the primary study eye. We also only included eyes with clear ocular media. BCVA had to be 20 ETDRS letters (20/400 Snellen equivalent) or better. The patient had to be able to cooperate in performing the examinations.

\section{DATA COLLECTION AND MANAGEMENT:}

Visual acuity information and demographic and clinical examination data were entered into REDCap and checked for completeness and consistency by the data coordinating center (DCC). ${ }^{19}$ The central reading center was the Doheny Imaging Reading Center (DIRC) at the Doheny Eye Institute. The coordinating and data management center was the Dana Center for Preventive Ophthalmology, Wilmer Eye Institute, Johns Hopkins University School of Medicine and Johns Hopkins Bloomberg School of Public Health. The Nidek MP-1 was used for all microperimetry tests according to the study protocol and all machines were certified by the DIRC. 6 
The quality and completeness of the submitted examinations was assessed by the DIRC and in case of poor-quality or missing data the centers were notified. Adjudication processes were applied if the 2 DIRC-certified graders had discordant gradings, and a final assessment was given by a DIRC investigator. All processed data were transferred electronically to the DCC.

\section{MICROPERIMETRY:}

Microperimetry and fixation measures were performed using the Nidek MP-1 microperimeter (Navis Software 1.7.0 or higher; Nidek Technologies Srl, Padova, Italy) using a modified Humphrey 10-2 grid as described in ProgStar Report No. 1.6 Dynamic quantification of fixation was performed during the sensitivity test. Fixation was also determined during a separate fixation task when the patients were asked to fixate their gaze steadily on the center of a red single cross on a white, monochromatic background. The extension of this cross was set as 2 degrees; the thickness was 1 degree as default, but both could be increased if necessary. All tests were performed monocularly with the contralateral eye patched. The duration for the fixation test was roughly 30 seconds after the patient had located the red cross. At a frequency of $25 \mathrm{~Hz}$, real-time fundus images were acquired and the locations of fixation were registered for each image. In order to reduce effects of learning, patients who never had an MP-1 examination before and for whom no scotopic examination was planned underwent a training examination before doing microperimetry. This training examination employed a different test pattern that included 8 test locations (white Goldmann III stimuli on a white background of 200 ms duration; 4-2 automatic strategy while fixating a red single cross of at least 2 degrees extension and 1 degree thickness).

\section{DESCRIPTION OF THE PREFERRED RETINAL LOCUS:}

The preferred retinal locus from the fovea (PRL) was defined as the distance in degrees between the center of gravity of all recorded fixation events and the foveal center. A concentric grid was automatically centered on the center of gravity of all recorded fixation points using Nidek's NAVIS software (Figure 1, Navis Software 1.7.0 or higher; Nidek Technologies Srl). Two graders at the central reading center manually determined the location of the anatomic fovea. For difficult cases with foveal atrophy, the graders looked for the point of maximal inner retinal layer convergence on optical coherence tomography and used the adjoining B-scans immediately superior and inferior to the approximate foveal center to determine this center as precisely as possible. In a preliminary subgroup analysis, 45 eyes were randomly selected and the PRL distance from the fovea was determined for the full macular sensitivity test and for the separate fixation examination using the described methods. The PRL eccentricity was not significantly different $\left(\rho_{\mathrm{RPL}}=0.87 ; P<.001 ; \mathrm{n}=\right.$ 45). We used fixation recordings from the macular sensitivity test to determine the PRL because this examination was available in a larger number of patients.

\section{DESCRIPTION OF THE BIVARIATE CONTOUR ELLIPSE AREA:}

The raw data containing the coordinates of each fixation event during the separate fixation task were extracted and the bivariate contour ellipse area (BCEA) was calculated using Microsoft Excel (Microsoft Corp, Redmond, Washington, USA). For this analysis, we used 
data from a separate fixation task as in most of the available literature on fixation stability. In this study, we calculated the global BCEA, which encompasses 1 standard deviation (SD) (68.3\%)-BCEA, of all fixation points (Figure 1, Left). The following equation was used to describe fixation stability: $B C E A=2 k \pi \sigma_{H} \sigma_{V}\left(1-\rho^{2}\right)^{1 / 2}$.

$\sigma_{H}$ and $\sigma_{V}$ are the standard deviations of the horizontal and vertical eye movements; $\rho$ is the Pearson product-moment correlation coefficient of eye movements in the horizontal and vertical meridian. $k$ is dependent on the chosen probability area $P=1-e^{-k}$ and $e$ is the base of the natural logarithm. For a probability of $68.3 \%, k$ must be about 1.15 for the equation to be true.

\section{STATISTICAL ANALYSES:}

Outcome measures for this manuscript include the eccentricity of the PRL from the anatomic fovea in degrees (deg) and the BCEA in degrees squared $\left(\mathrm{deg}^{2}\right)$. Linear models were used to estimate the association between the 1-year change in PRL eccentricity and the 1-year change in BCEA, to account for the correlation between eyes of the same subject. Standard errors were corrected using the generalized estimated equation approach. Linear mixed models with time as the independent variable were used to estimate the yearly change for each outcome.

\section{RESULTS}

AT BASELINE, MP-1 WAS GRADED FOR A TOTAL OF 238 Patients (444 eyes). The PRL was determined for 396 eyes and was ungradable for 48 eyes (12 eyes with the test terminated before completion, 26 with missing color fundus images, 3 eyes with multiple PRLs, 7 with other problems). The BCEA was determined for 427 eyes at baseline; 17 eyes had missing values (13 without a fixation test performed, 3 eyes with technical problems, and 1 eye with another reason). At the month 12 visit, MP-1 was graded for 218 patients (408 eyes). For the month 12 visit, the PRL was determined for 392 eyes and was ungradable for 16 eyes ( 6 eyes with the test terminated before completion, 3 with missing color fundus images, 6 where the grader was unable to determine the foveal center, 1 eye with other problems). The BCEA was determined for 344 eyes at baseline with 64 ungradable BCEAs (fixation examination not performed in 60 eyes, and 4 eyes with technical problems). Both the baseline and month 12 visits were gradable for the PRL in $79 \%$ of eyes and for the BCEA in $74 \%$ of eyes. The complete enrollment count is detailed in the Supplemental Table (Supplemental Material available at AJO.com).

Table 1 shows the demographic features of the cohort at baseline. Out of a total of 238 patients at baseline, 105 were male (44\%), 203 were white (85\%), and all had a molecularly confirmed diagnosis of STGD1. The median age of the participant cohort was 32 years (mean \pm SD, $33.8 \pm 15.2$ years; range, 7-69 years). Out of 238 patients, $100(42 \%)$ had first symptoms of macular disease at age 18 years or earlier and 121 patients (51\%) developed symptoms later. Only 2 participants (1\%) were diagnosed with STGD1 without symptoms and 15 patients $(6 \%)$ did not recall the beginning of their symptoms. Overall, there were 444 eligible eyes; 206 participants (86.6\%) contributed with 2 eyes and 32 participants (13.4\%) with 1 eye. Median BCVA, evaluated in the 444 eyes at baseline, was 42 ETDRS letters 
(mean, 46.2 \pm 16.3 ETDRS letters; range, 20-88 letters; the lower limit of 20 ETDRS letters for the BCVA is based on the inclusion criteria of the ProgStar study).

The distance of the PRL, which is defined as the gravitational center of all fixation points from the fovea, was graded in 396 eyes at baseline, in 386 eyes at 6 months, and in 392 eyes at 12 months. Data from the separate fixation test to evaluate the stability of fixation are available for 412 eyes at baseline, for 336 eyes at 6 months, and for 333 eyes at 12 months. The measurements for the eccentricity of the PRL and the BCEA for the examinations at baseline and at 6 and 12 months of follow-up are presented in Table 2. After 12 months of follow-up, the change in the eccentricity of the PRL from the anatomic fovea was -0.0014 $\operatorname{deg}(95 \%$ confidence interval $[\mathrm{CI}],-0.27 \mathrm{deg}, 0.27 \mathrm{deg} ; P=.99)$. The deterioration in the stability of fixation as expressed by a larger BCEA encompassing $1 \mathrm{SD}$ of all fixation points was $1.21 \mathrm{deg}^{2}\left(95 \% \mathrm{CI},-1.23 \mathrm{deg}^{2}, 3.65 \mathrm{deg}^{2} ; P=.33\right.$ ). The estimates of the yearly rate of change of fixation parameters are presented in Table 3. One degree of additional PRL eccentricity after 12 months was associated with an increase of $0.89 \mathrm{deg}^{2}$ in BCEA after 12 months, but this correlation did not reach statistical significance $(P=.14, \mathrm{n}=276$, Figure 2$)$.

There were individual cases where the fixation parameters delivered interesting information about an eye's visual function that could not be captured with other imaging or functional equipment. Figure 3 shows the example of an eye in which fixation first turned to a more central location (from $4.5 \mathrm{deg}$ to $3.0 \mathrm{deg}$ distance from the fovea) before it began to fixate with a more eccentric location ( $6.0 \mathrm{deg}$ from the fovea). Against the trend in the overall cohort, fixation also became more stable this case $\left(\mathrm{BCEA}_{\text {baseline }}=4.82 \mathrm{deg}^{2} ; \mathrm{BCEA}_{\mathrm{Month} 12}\right.$ $=2.21 \operatorname{deg}^{2}$ ).

\section{DISCUSSION}

THIS RESEARCH PROVIDES DATA ON THE LONGITUDINAL changes of fixation parameters assessed with the Nidek MP-1 microperimeter in a large cohort of patients with molecularly confirmed STGD1. Using continuous parameters to quantify fixation location and stability in cross-sectional data, we recently showed that an earlier age of onset of STGD1 symptoms and poor BCVA are associated with more unstable and more eccentric fixation. ${ }^{12}$ Hence, our previous results suggested that fixation may be a useful visual function biomarker. However, there is only little data on longitudinal changes using microperimetry. ${ }^{18}$ Furthermore, most of the available literature on fixation assessment used noncontinuous, less accurate categories, or they simply localized the fixation cloud to a certain quadrant of the posterior pole, ${ }^{14,20-23}$ for example, foveal, parafoveal, and extrafoveal. ${ }^{18}$ To the best of our knowledge, this is the first report on prospective longitudinal changes of the PRL eccentricity and fixation stability using the BCEA in STGD1.

\section{MONTH 12 CHANGES IN FIXATION LOCATION:}

The distance of the center of all fixation events from the anatomic fovea in degrees was determined during the macular sensitivity test for all eyes and a median of 6.0 degrees was found at baseline. In a preparatory study comparing fixation location in 45 eyes with STGD1 obtained during a separate fixation task with data from the macular threshold test, we found 
no difference in eccentricity from the fovea $\left(\rho_{\mathrm{RPL}}=0.87 ; P<.001 ; \mathrm{n}=45\right)$. Therefore, we used the data from the macular threshold test to determine the eccentricity of the PRL. After 12 months of follow-up, the statistical analysis showed no change in the eccentricity of fixation of 0.0014 degrees (95\% CI: $-0.27 \mathrm{deg}, 0.27 \mathrm{deg} ; P=.99$ ). Based on the association of longer disease duration with more eccentric fixation in our own cohort ${ }^{12}$ and in a report by Testa and associates, ${ }^{18}$ we expected the fixation location to represent the progressive nature of the disease and to become more eccentric during follow-up. Multiple reasons may account for the observed null change. Firstly, 12 months of follow-up may not be enough to detect a significant change using this method. Secondly, there is a large heterogeneity in the pattern of the development of fixation location with eyes where fixation turned from a very eccentric location toward the fovea and eyes where fixation became more eccentric, overall outweighing the change. Certain subgroups based on baseline fixation may show a unidirectional change. Thirdly, some eyes may actually have multiple PRLs with an unknown effect on the analysis. Fourth, there may be adaptation processes with an improvement of fixation that allow more central fixation.

\section{MONTH 12 CHANGES IN FIXATION STABILITY:}

We used the BCEA as a continuous parameter to describe how close the fixation points are together, as opposed to other study groups that used the percentage of fixation points within a 2- or 4-degree circle or the Fujii categorization. ${ }^{18,22}$ The BCEA as a continuous parameter has been shown to have multiple advantages over the semi-quantitative Fujii method, such as a better correlation with reading abilities, the absence of arbitrary categorizations, and the recognition of the elliptical distribution of the registered fixation events. ${ }^{24,25}$ Using the Nidek MP-1, the repeatability of the estimated BCEA has been shown to be acceptable. ${ }^{26}$ The ability to deliberately fixate a target was tested during a short fixation task because longer testing durations (as during the sensitivity test) may destabilize fixation. ${ }^{27}$ For the baseline cohort, we found a mean \pm SD of the 1 SD-BCEA of $4.5 \pm 9.1 \mathrm{deg}^{2}$ (median [interquartile range], $2.3 \mathrm{deg}^{2}$ [0.8, $4.7 \mathrm{deg}^{2}$ ]; range, $0.01 \mathrm{deg}^{2}, 134.9 \mathrm{deg}^{2}$ ). Values of the 1 SD-BCEA between $0.08 \mathrm{deg}^{2}$ and $0.45 \mathrm{deg}^{2}$ can be found in the literature for nondiseased eyes. ${ }^{27-29}$ We tested again after 6 and 12 months of follow-up and calculated a yearly change of $1.21 \mathrm{deg}^{2}$ in 1 SD-BCEA (95\% CI: $-1.23 \mathrm{deg}^{2}, 3.65 \mathrm{deg}^{2} ; P=.33$ ). We recently reported an association of longer disease duration with more unstable fixation in STGD1. ${ }^{12}$ We therefore expected an increase in BCEA during follow-up. The direction of the change is as predicted and may turn statistically significant in the month 24 data of the ProgStar study. Many eyes demonstrated a diminished size of the BCEA after 12 months, suggesting possible neuronal adaptation processes with an unknown magnitude of effect. The literature provides some evidence for improvement of fixation through eccentric viewing training in patients with macular disease. ${ }^{30,31}$ Similar processes may have taken place in our study population, but although many eyes showed more stable fixation after 12 months, the overall mean BCEA increased and remained far larger than for nondiseased eyes. ${ }^{27-29}$ Moreover, the presence of multiple PRLs, which are present in about $5 \%$ of eyes with STGD1, ${ }^{20}$ may have altered our measurements because we used 1 global BCEA to describe fixation stability. One limitation to our results is the fact that the separate fixation test was not performed in a number of cases. This led to a loss of BCEA values on follow-up. 


\section{CONCLUSIONS}

\section{THIS IS THE FIRST PROSPECTIVE LONGITUDINAL ANALYSIS of continuous} fixation parameters in a large cohort of genetically confirmed cases of $A B C A 4$-related STGD1. The results may help better understand the complex nature of fixation measures and how they change over time. Based on the presented results, a follow-up period of 12 months does not provide statistically significant changes for a large cohort of patients that could be used to compare effects of treatments in clinical trials. Underlying reasons may be the complex heterogeneity of the changes of fixation parameters that include both deteriorations and improvements. Neuronal adaptation processes may be involved in cases where fixation becomes more central or more stable over time. It is also possible that a follow-up period of 12 months is too short a period to demonstrate significant centrifugal displacement and destabilization of fixation despite the large number of study participants. However, fixation parameters may serve as useful secondary outcome parameters in selected cases and for counseling patients to explain changes to their visual functionality. In addition, the observed changes may explain cases of improvement in BCVA.

\section{Supplementary Material}

Refer to Web version on PubMed Central for supplementary material.

\section{Other Acknowledgments:}

These results were published in part at the Association for Research in Vision and Ophthalmology (ARVO), as follows: Schönbach EM: Cross-sectional evaluation of microperimetric fixation location and stability in Stargardt disease in the ProgStar study (abstract 4635). Annual ARVO Meeting, Baltimore, May 2017; Schönbach EM: Month 24 changes in microperimetric mean sensitivity, fixation location, and fixation stability in Stargardt disease: The ProgStar Study (abstract 2694). Annual ARVO Meeting, Seattle, May 2016; Khan S: Twelve-month-change in microperimetric mean sensitivity, fixation location and fixation stability in Stargardt disease: The prospective ProgStar study (abstract 4286). Annual ARVO Meeting, Seattle, May 2016.

FUNDING/SUPPORT: THE PROGSTAR STUDIES ARE SUPPORTED BY THE FOUNDATION FIGHTING BLINDNESS (FFB) CLINICAL Research Institute (Columbia, Maryland, USA) and a grant by the U.S. Department of Defense USAMRMC TATRC to FFB (Fort Meade, Maryland, USA; grant numbers W81XWH-07-1-0720 and W81-XWH-09-2-0189). Rupert W. Strauss is supported by the Austrian Science Fund (Vienna, Austria; FWF; Project number: J 3383-B23) and the Foundation Fighting Blindness Clinical Research Institute (Columbia, Maryland, USA). Etienne M. Schönbach is supported by the German National Academy of Sciences Leopoldina, Grant Number LPDS 2015-14 (Halle, Germany) and the Foundation Fighting Blindness Clinical Research Institute (Columbia, Maryland, USA). Financial Disclosures: Etienne M. Schönbach is supported by the German National Academy of Sciences Leopoldina, Grant Number LPDS 2015-14 (Halle, Germany) and the Foundation Fighting Blindness Clinical Research Institute (Columbia, Maryland, USA). Rupert W. Strauss is supported by the Austrian Science Fund (Vienna, Austria; FWF; Project number: J 3383-B23) and the Foundation Fighting Blindness Clinical Research Institute (Columbia, Maryland, USA). Janet S. Sunness is a consultant for Genentech (San Francisco, California, USA) and is on the scientific advisory board of Acucela (Tokyo, Japan) and Apellis (Crestwood, Kentucky, USA) and on the data safety and monitoring committee for Cell Cure's OpRegen study (Jerusalem, Israel). David G. Birch is a consultant for AGTC (Alachua, Florida, USA), Ionis (Carlsbad, California, USA), Genentech (South San Francisco, California, USA), Nightstar (London, England), and Nacuity (Ft. Worth, Texas, USA), and is supported by the Foundation Fighting Blindness Clinical Research Institute (FFB CRI, Columbia, Maryland). SriniVas R. Sadda is financially supported by Allergan (Dublin, Ireland), Carl Zeiss Meditec (Jena, Germany), Genentech (San Francisco, California, USA), and Optos (Dunfermline, UK), and is a consultant for Allergan (Dublin, Ireland), CenterVue (Padova, Italy), Genentech (San Francisco, California, USA), Heidelberg Engineering (Heidelberg, Germany), Iconic Therapeutics, Inc (San Francisco, California, USA), NightstarX (London, UK), Novartis (Basel, Switzerland), Optos (Dunfermline, UK), Thrombogenics (Leuven, Belgium), and Topcon (Tokyo, Japan). Sheila K. West is a scientific technical advisory committee member for the Alcon Research Institute (Fort Worth, Texas), and for Research to Prevent Blindness (New York, New York). Dr. Scholl is a paid consultant of the following entities (not including the National Institutes of Health): Fovea Pharmaceuticals/Sanofi (Paris, France); Trevena Inc. (Chesterbrook, PA); Guidepoint Global, LLC (New York, 
NY); Gerson Lehrman Group (New York, NY); Shire (Dublin, Republic of Ireland). Dr. Scholl is a member of the Scientific Advisory Board of Vision Medicines, Inc. Hendrik P.N. Scholl is a member of the Data Monitoring Committee of the following entities (not including the National Institutes of Health): Genentech Inc (San Francisco, California, USA)/F. Hoffmann-La Roche Ltd (Basel, Switzerland); StemCells, Inc (Newark, California, USA); Genzyme Corp (Boston, Massachusetts, USA)/Sanofi (Paris, France); he also serves as a member of the Ophthalmic Devices Panel of the Medical Devices Advisory Committee, Food and Drug Administration (FDA, Silver Spring, Maryland, USA). These arrangements have been reviewed and approved by the Johns Hopkins University in accordance with its conflict of interest policies. Hendrik P.N. Scholl is principal investigator of grants at The Johns Hopkins University sponsored by the following entity (not including the National Institutes of Health): QLT, Inc (Vancouver, Canada). Grants to investigators at Johns Hopkins University are negotiated and administered by the institution (such as the School of Medicine) which receives the grants, typically through the Office of Research and Administration. Individual investigators who participate in the sponsored project(s) are not directly compensated by the sponsor but may receive salary or other support from the institution to support their effort on the project(s). The following authors have no financial disclosures: Xiangrong Kong, Beatriz Muñoz, Mohamed A. Ibrahim, GesaAstrid Hahn, Fadi Nasser, and Eberhart Zrenner. All authors attest that they meet the current ICMJE criteria for authorship.

\section{REFERENCES}

1. Walia S, Fishman GA. Natural history of phenotypic changes in Stargardt macular dystrophy. Ophthalmic Genet 2009;30(2): 63-68. [PubMed: 19373676]

2. Tanna P, Strauss RW, Fujinami K, Michaelides M. Stargardt disease: clinical features, molecular genetics, animal models and therapeutic options. Br J Ophthalmol 2017;101(1):25-30. [PubMed: 27491360]

3. Fishman GA, Farber M, Patel BS, Derlacki DJ. Visual acuity loss in patients with Stargardt's macular dystrophy. Ophthalmology 1987;94(7):809-814. [PubMed: 3658351]

4. Scholl HP, Strauss RW, Singh MS, et al. Emerging therapies for inherited retinal degeneration. Sci Transl Med 2016; 8(368):368rv6.

5. Scholl HP, Sahel JA. Gene therapy arrives at the macula. Lancet 2014;383(9923):1105-1107. [PubMed: 24439295]

6. Strauss RW, Ho A, Munoz B, et al. The Natural History of the Progression of Atrophy Secondary to Stargardt Disease (ProgStar) studies: design and baseline characteristics: ProgStar Report No. 1. Ophthalmology 2016;123(4):817-828. [PubMed: 26786511]

7. Kong X, West SK, Strauss RW, et al. Progression of Visual Acuity and Fundus Autofluorescence in Recent-Onset Stargardt Disease: ProgStar Study Report \#4. Ophthalmol Retina 2017;1(6):514-523. [PubMed: 31047445]

8. Strauss RW, Munoz B, Ho A, et al. Incidence of atrophic lesions in Stargardt disease in the Progression of Atrophy Secondary to Stargardt Disease (ProgStar) study: Report No. 5. JAMA Ophthalmol 2017;135(7):687-695. [PubMed: 28542697]

9. Kong X, Strauss RW, Michaelides M, et al. Visual acuity loss and associated risk factors in the retrospective Progression of Stargardt Disease Study (ProgStar Report No. 2). Ophthalmology 2016;123(9):1887-1897. [PubMed: 27378015]

10. Kong X, Strauss RW, Cideciyan AV, et al. Visual acuity change over 12 months in the prospective Progression of Atrophy Secondary to Stargardt Disease (ProgStar) study: ProgStar Report Number 6. Ophthalmology 2017;124(11): 1640-1651. [PubMed: 28549516]

11. Schönbach EM, Wolfson Y, Strauss RW, et al. Macular sensitivity measured with microperimetry in Stargardt disease in the Progression of Atrophy Secondary to Stargardt Disease (ProgStar) study: Report No. 7. JAMA Ophthalmol 2017; 135(7):696-703. [PubMed: 28542693]

12. Schönbach EM, Ibrahim MA, Strauss RW, et al. Fixation location and stability using the MP1 microperimeter in Stargardt disease: ProgStar Report No. 3. Ophthalmol Retina 2016; 1(1):68-76. [PubMed: 31047397]

13. Crossland MD, Culham LE, Rubin GS. Fixation stability and reading speed in patients with newly developed macular disease. Ophthalmic Physiol Opt 2004;24(4):327-333. [PubMed: 15228511]

14. Sunness JS, Applegate CA, Haselwood D, Rubin GS. Fixation patterns and reading rates in eyes with central scotomas from advanced atrophic age-related macular degeneration and Stargardt disease. Ophthalmology 1996; 103(9):1458-1466. [PubMed: 8841306] 
15. Nilsson UL, Frennesson C, Nilsson SE. Location and stability of a newly established eccentric retinal locus suitable for reading, achieved through training of patients with a dense central scotoma. Optom Vis Sci 1998;75(12): 873-878. [PubMed: 9875992]

16. Nilsson UL, Frennesson C, Nilsson SE. Patients with AMD and a large absolute central scotoma can be trained successfully to use eccentric viewing, as demonstrated in a scanning laser ophthalmoscope. Vision Res 2003;43(16):1777-1787. [PubMed: 12818347]

17. Plank T, Frolo J, Brandl-Ruhle S, et al. Gray matter alterations in visual cortex of patients with loss of central vision due to hereditary retinal dystrophies. NeuroImage 2011; 56(3):1556-1565. [PubMed: 21352929]

18. Testa F, Melillo P, Di Iorio V, et al. Macular function and morphologic features in juvenile Stargardt disease: longitudinal study. Ophthalmology 2014;121(12):2399-2405. [PubMed: 25097154]

19. Harris PA, Taylor R, Thielke R, Payne J, Gonzalez N, Conde JG. Research electronic data capture (REDCap)-a metadata-driven methodology and workflow process for providing translational research informatics support. J Biomed Inform 2009;42(2):377-381. [PubMed: 18929686]

20. Reinhard J, Messias A, Dietz K, et al. Quantifying fixation in patients with Stargardt disease. Vision Res 2007;47(15): 2076-2085. [PubMed: 17562343]

21. Rohrschneider K, Gluck R, Blankenagel A, Volcker HE. Fixation behavior in Stargardt disease. Fundus-controlled studies. Ophthalmologe 1997;94(9):624-628. [PubMed: 9410227]

22. Plank T, Frolo J, Farzana F, Brandl-Ruhle S, Renner AB, Greenlee MW. Neural correlates of visual search in patients with hereditary retinal dystrophies. Hum Brain Mapp 2013; 34(10):2607-2623. [PubMed: 22505353]

23. Cideciyan AV, Swider M, Aleman TS, et al. ABCA4-associated retinal degenerations spare structure and function of the human parapapillary retina. Invest Ophthalmol Vis Sci 2005; 46(12):4739-4746. [PubMed: 16303974]

24. Fujii GY, de Juan E Jr, Sunness J, Humayun MS, Pieramici DJ, Chang TS. Patient selection for macular translocation surgery using the scanning laser ophthalmoscope. Ophthalmology 2002;109(9):1737-1744. [PubMed: 12208725]

25. Crossland MD, Dunbar HM, Rubin GS. Fixation stability measurement using the MP1 microperimeter. Retina 2009; 29(5):651-656. [PubMed: 19262440]

26. Bedell HE, Pratt JD, Krishnan A, et al. Repeatability of Nidek MP-1 fixation measurements in patients with bilateral central field loss. Invest Ophthalmol Vis Sci 2015;56(4):2624-2630. [PubMed: 25788656]

27. Longhin E, Convento E, Pilotto E, et al. Static and dynamic retinal fixation stability in microperimetry. Can J Ophthalmol 2013;48(5):375-380. [PubMed: 24093183]

28. Dunbar HM, Crossland MD, Rubin GS. Fixation stability: a comparison between the Nidek MP-1 and the Rodenstock scanning laser ophthalmoscope in persons with and without diabetic maculopathy. Invest Ophthalmol Vis Sci 2010;51(8): 4346-4350. [PubMed: 20335616]

29. Rigoni ECC, Cacciotti V, Trabucco P. Normal values and repeatability of bivariate contour ellipse area (BCEA) in microperimeter MP-1. Invest Ophthalmol Vis Sci 2014; 155(3):169.

30. Tarita-Nistor L, Gonzalez EG, Mandelcorn MS, Lillakas L, Steinbach MJ. Fixation stability, fixation location, and visual acuity after successful macular hole surgery. Invest Ophthalmol Vis Sci 2009;50(1):84-89. [PubMed: 18757517]

31. Crossland MD, Culham LE, Kabanarou SA, Rubin GS. Preferred retinal locus development in patients with macular disease. Ophthalmology 2005;112(9):1579-1585. [PubMed: 16087239] 

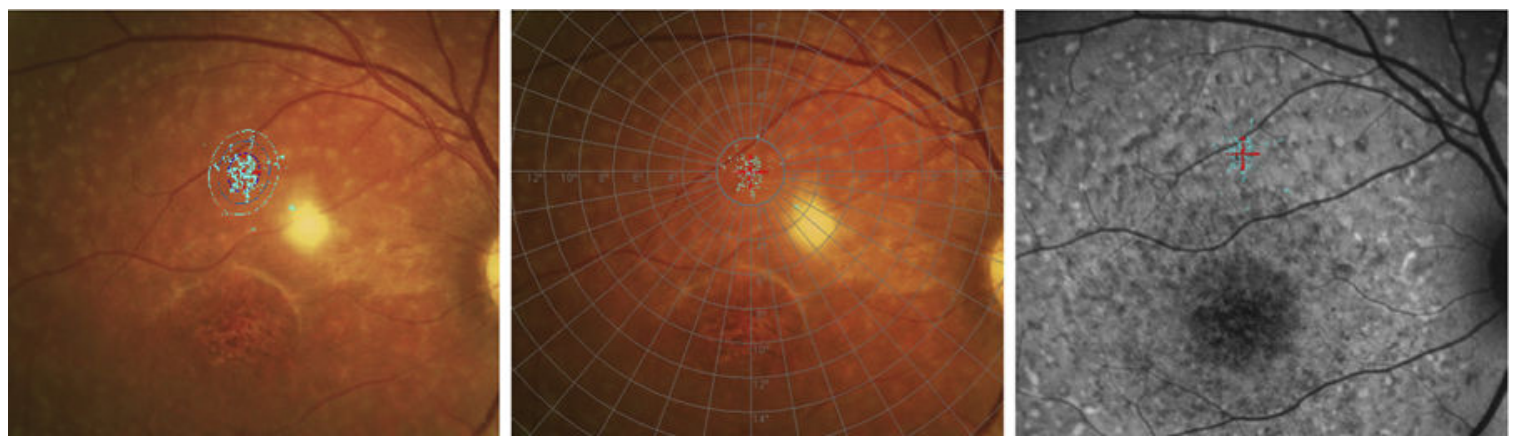

FIGURE 1.

Grading of the bivariate contour ellipse area (BCEA), the preferred retinal locus, and analysis of fixation on fundus autofluorescence images in the example of the right eye of an 18-year-old woman who had been diagnosed with Stargardt disease 5 years before. (Left) The BCEA is the smallest ellipse that encompasses 1, 2, or 3 standard deviations of all fixation events. The MP-1 microperimeter can fully automatically draw this ellipse around the recorded fixation points and calculate the area as shown in the image. A smaller BCEA corresponds to more stable fixation. (Center) A grid was automatically placed on the center of gravity of all fixation events in blue color. The distance between the center of the grid and the fovea could then be determined. (Right) The recorded fixation events can be overlaid on a fundus autofluorescence image, which allows for clearer identification of atrophic macula and analysis of fixation in relation to atrophy. 


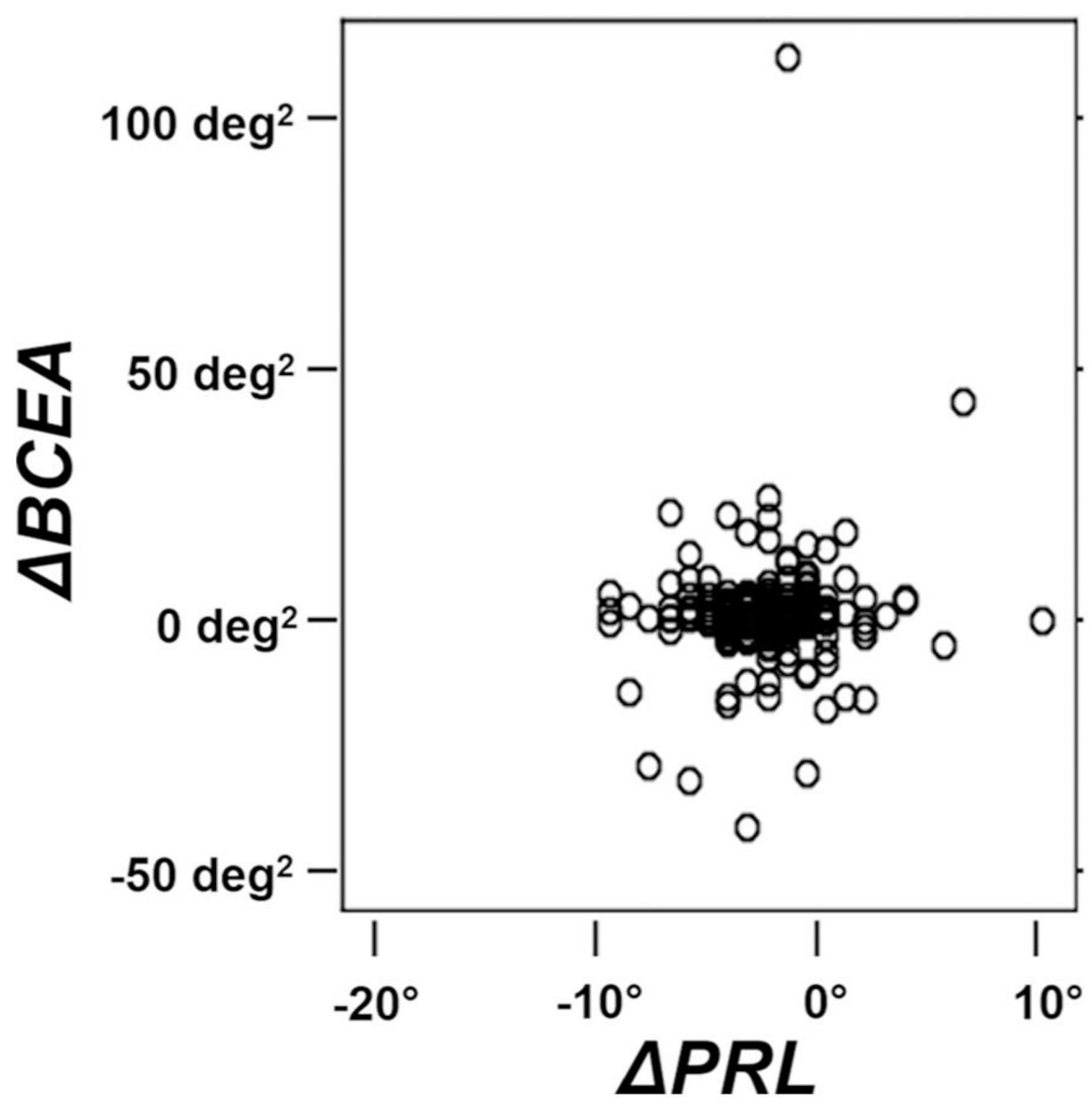

FIGURE 2.

Correlation of the change in eccentricity of the preferred retinal locus (PRL) with the change in 1 SD-BCEA (bivariate contour ellipse area using 1 standard deviation). Each degree of additional PRL eccentricity was associated with a 1 SD-BCEA, which is $0.089 \mathrm{deg}^{2}$ larger $(P=.14 ; \mathrm{n}=276)$. 

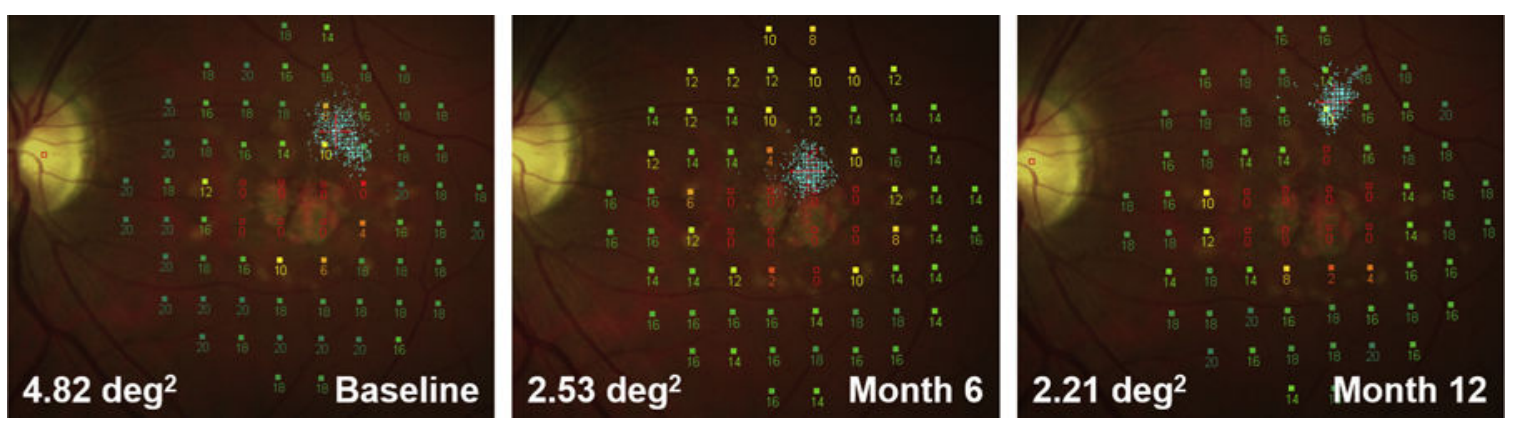

FIGURE 3.

Example of a left eye of a 45-year-old man 14 years after diagnosis that first developed more central, then more eccentric fixation over the course of 12 months. In this example, fixation becomes more stable on each follow-up visit. This case demonstrates the complex nature of the course of fixation parameters over time and how they may provide useful additional information on top of structural or other functional parameters. 
TABLE 1.

Demographic Features for All Enrolled Patients ( $\mathrm{N}=238)$ at Baseline

\begin{tabular}{lc}
\hline Feature & Result \\
\hline Sex, $\mathrm{n}(\%)$ & \\
Male & $105(44.1 \%)$ \\
Female & $133(55.9 \%)$ \\
Race, $\mathrm{n}(\%)$ & \\
Asian/South Asian & $10(4.2 \%)$ \\
African American & $20(8.4 \%)$ \\
Unknown & $4(1.7 \%)$ \\
Hybrid ethnicity & $1(0.4 \%)$ \\
White & $203(85.4 \%)$ \\
Age of disease onset, $\mathrm{n}(\%)$ & \\
$\leq 18$ & $100(42.0 \%)$ \\
$>18$ & $121(50.8 \%)$ \\
Asymptomatic & $2(0.84 \%)$ \\
Unknown & $15(6.3 \%)$ \\
Current age (y), median (mean \pm SD, range) & $32(33.75 \pm 15.19,7-69)$ \\
Age of onset (y), median (mean \pm SD, range) & $20(22.57 \pm 12.84,4-64)$ \\
Duration of disease (y), median (mean \pm SD, range) & $9(11.77 \pm 9.47,0-55)$ \\
Eligible eyes per participant, $\mathrm{n}(\%)$ & \\
Bilateral & $206(86.6 \%)$ \\
Unilateral & $32(13.4 \%)$ \\
Best-corrected visual acuity (ETDRS letters, N = 444 eyes), median (mean $\pm \mathrm{SD}$, range) & $42(46.2 \pm 16.3,20-88)$ \\
\hline
\end{tabular}

ETDRS $=$ Early Treatment Diabetic Retinopathy Study. 


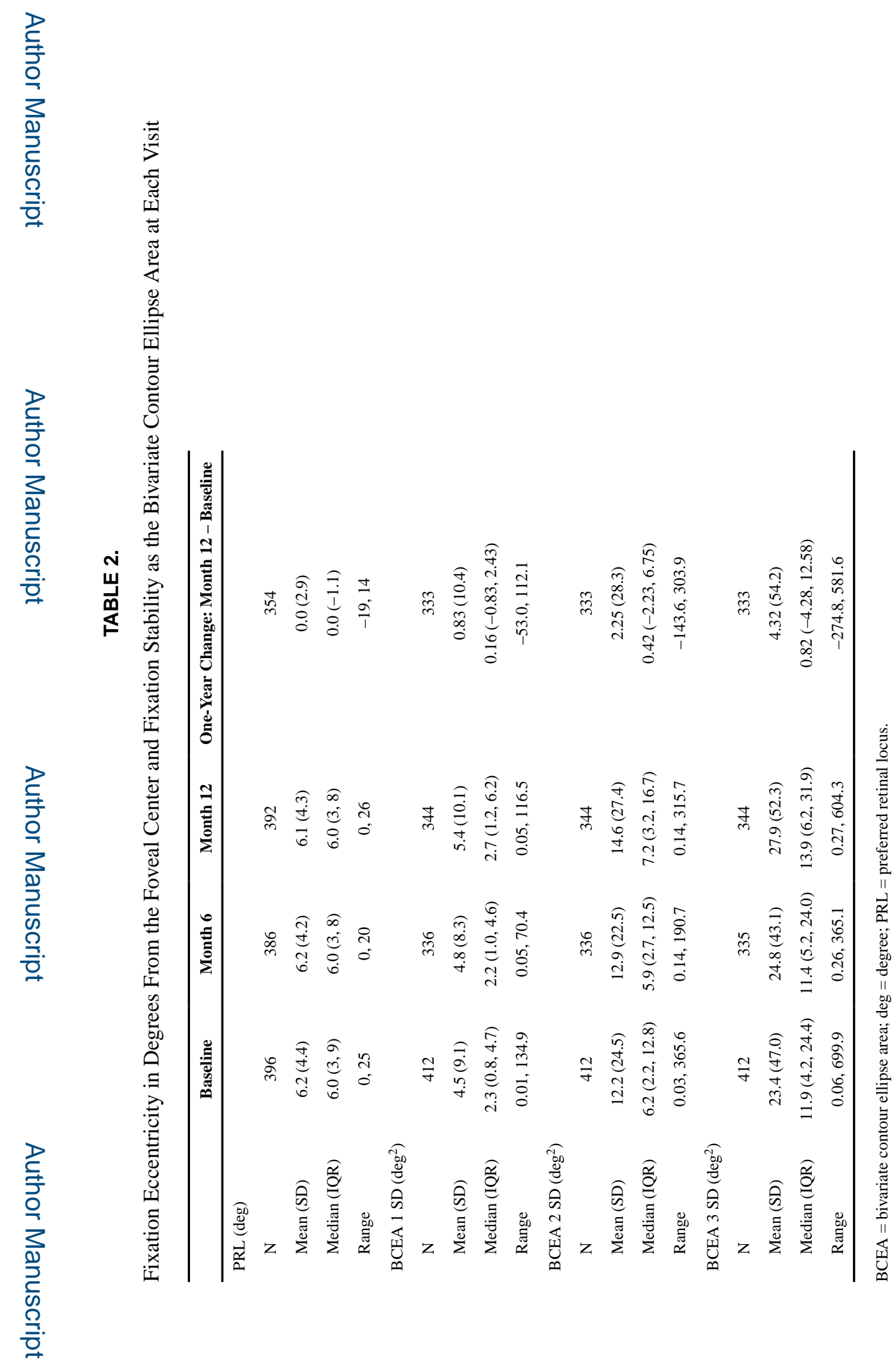


TABLE 3.

Mixed Linear Models Considering All 3 Points in Time: Estimated Yearly Changes in Microperimetric Parameters Using Mixed Linear Models

\begin{tabular}{lccc}
\hline & Yearly Increase & $\boldsymbol{P}$ & $\mathbf{9 5 \%}$ CI \\
\hline PRL (deg) & -0.0014 & .99 & $-0.27+0.27$ \\
BCEA 1 SD $\left(\mathrm{deg}^{2}\right)$ & 1.21 & .33 & $-1.23+3.65$ \\
BCEA 2 SD $\left(\mathrm{deg}^{2}\right)$ & 3.67 & .27 & $-2.91+10.26$ \\
BCEA 3 SD $\left(\mathrm{deg}^{2}\right)$ & 6.93 & .28 & $-5.70+19.57$ \\
\hline
\end{tabular}

$\mathrm{BCEA}=$ bivariate contour ellipse area; deg $=$ degree $; \mathrm{PRL}=$ preferred retinal locus. 